\title{
Effect of Multiple Orders on Superconducting Transition Temperature of Hole Doped Cuprates
}

\author{
I. Qabid, S. H. Naqib* \\ Department of Physics, University of Rajshahi, Rajshahi 6205, Bangladesh \\ Received 15 May 2017, accepted in final revised form 21 September 2017
}

\begin{abstract}
Hole doped high- $T_{\mathrm{c}}$ cuprate superconductors are strongly correlated electronic systems. In these materials, various electronic orders are often found, but whether they support or compete with superconducting order is not unambiguous. Superconductivity normally manifests itself by a superconducting gap in the electronic density of states (EDOS). In cuprates, a gap appears even in the normal state called the pseudogap (PG). For certain doping range, spin density wave and charge density wave coexist with superconductivity by inducing corresponding additional gaps in the EDOS. In this study, we have tried to obtain expression for superconducting transition temperature, $T_{\mathrm{c}}$ by solving the BCS (Bardeen-Cooper-Schrieffer) energy gap equation in the presence of depleted EDOS of various origins and types. We have been successful to solve the weak-coupling BCS integral equation analytically in some special cases and also in the general case by using numerical integration. We have found that depending on conditions these non-pairing gaps/orders can enhance as well as reduce $T_{\mathrm{c}}$.
\end{abstract}

Keywords: Superconductivity; Cuprates; Multiple orders; Electronic density of states.

(C) 2017 JSR Publications. ISSN: 2070-0237 (Print); 2070-0245 (Online). All rights reserved. doi: http://dx.doi.org/10.3329/jsr.v9i4.32598 J. Sci. Res. 9 (4), 341-349 (2017)

\section{Introduction}

Research in superconductivity (SC) aims at synthesizing room temperature ( $300 \mathrm{~K}$ ) superconductors and to understand the mechanism leading to high- $T_{c}$. Our interest in cuprates is due to the fact that they have been the only type offering the highest $T_{c}$ at ambient pressure and are very rich in physics [1]. Understanding the electronic phase diagram of cuprates temperature and doping dependence of generic physical properties (Fig. 1), is central to understand the cuprate systems as a whole.

At low temperature and doping $(p)$, the cuprates are antiferromagnetic (AF) insulators. There is a region called the spin glass (SG) region (not present in all the cuprates; not shown in Fig. 1) which exists at low temperature between the AF and superconducting phases for some cuprates (e.g. in LSCO and in BSCO, but not in

* Corresponding author: salehnaqib@yahoo.com 
YBCO). This is a region of short range magnetic order due to freezing of spins below a certain temperature, $T_{g}$ (SG temperature). Cuprates superconduct below a critical temperature, $T_{c}$, within the doping level $0.05<p<0.27$, with $T_{c}$ varying approximately parabolically with hole doping given by an empirical formula [3]

$T_{c}(p)=T_{c}^{\max }\left[1-82.6(p-0.16)^{2}\right]$

where, $T_{c}^{\max }$ is the maximum $T_{c}$ at optimum doping $(p \sim 0.16)$.

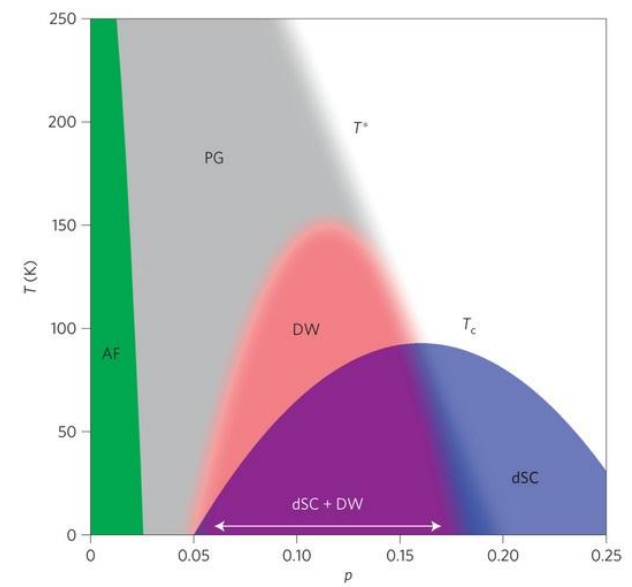

Fig. 1. Electronic $T$ - $p$ phase diagram of cuprates [2].

The overdoped (OD) region $(p>0.16)$ of the phase diagram is often called the Fermi-liquid (F-L) region. In this region, the properties of single electrons are renormalized by interactions with other electrons to form quasiparticles. The part of the phase diagram between the underdoped (UD) and Fermi liquid regions and above the optimally doped region, is called the non-Fermi-liquid (non-F-L) region. The thermodynamic properties in this region are similar to the behavior of an F-L whereas, the transport properties have exceptionally simple but unusual power laws as a function of temperature. In the normal state of the UD cuprates, pseudogap (PG) appears at the Fermi energy below a certain temperature $T^{*}$, known as the PG temperature. Almost all the physical properties of cuprates are affected by the presence and magnitude of the PG [4]. The PG manifests itself in various thermodynamic, transport, and magnetic properties. For instance, the cross-over from the linear dependence of the resistivity on temperature in the non-F-L region to a stronger dependence in the PG region is a signature of the PG. Some researchers opine that $T^{*}$ extends to the superconducting region up to a quantum critical point (QCP), the point in the phase diagram of a material where a continuous phase transition occurs at absolute zero due to quantum fluctuations caused by Heisenberg's uncertainty principle [5,6] ( $p \sim 0.19$, in the $T-p$ phase diagram). 
There is a gap in the EDOS at the Fermi level due to SC. In the underdoped region, an additional gap, the pseudogap [7] appears even in the normal state. If spin and charge density orders are present, then they also introduce gaps. Whether these gaps have anything to do with the superconducting gap is not clear yet. All hole doped cuprates show PG, charge density wave (CDW) and spin density wave (SDW) $[8,9]$ orders for certain range of hole concentrations. In this short communication, we investigate the underlying correlation among these density waves and superconducting pairing by studying the effects of the non-superconducting gaps on $T_{c}$. For this, we have started with the Eliashberg theory for superconductivity, which for the weak coupling and zero Coulomb pseudopotential reduces to the BCS gap integral. Using this weak coupling BCS integral, attempts have been made to find expressions for $T_{\mathrm{c}}$ for various conditions. From the nature of $T_{\mathrm{c}}$, the correlation among various orders and superconductivity has been examined. This paper has been organized as follows - we have presented the theoretical formalism and analysis in Section 2. Section 3 consists of discussion and conclusions.

\section{Theoretical Formalism and Analysis}

We have the BCS $T_{c}$ equation

$$
\frac{2}{g}=\int_{-\omega_{c}}^{\omega_{c}} N(\varepsilon) \frac{1}{\varepsilon} \tanh \frac{\varepsilon}{2 T_{c}} d \varepsilon
$$

where, $g$ is the pairing potential and $\omega_{c}$ is the bosonic cut-off energy.

PG, most probably, does not have explicit temperature dependence [10], while CDW and SDW gaps are believed to have temperature dependences [11] but no explicit form is known yet. Thus, for simplicity, we will assume that the nonsuperconducting gaps are temperature independent. We have taken the form of the gap to be triangular so that $N(\varepsilon)$ takes the form shown in Fig. 2 .

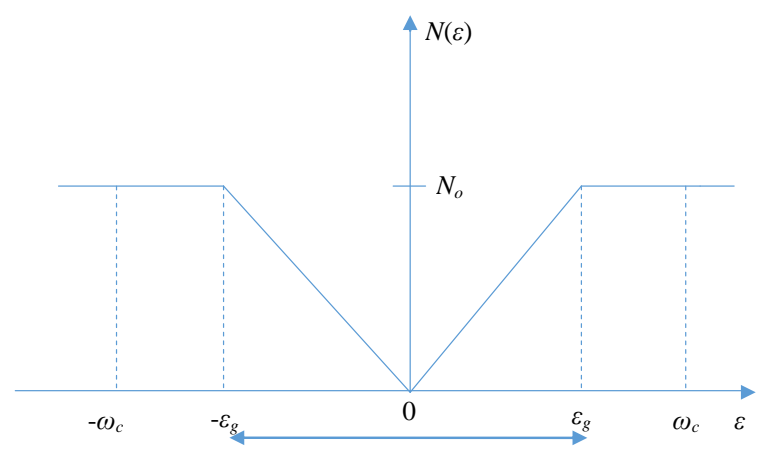

Fig. 2. EDOS in the presence of a (triangular) gap. 
$N(\varepsilon)=N_{o} ; \quad|\varepsilon|>\varepsilon_{g}$

$N(\varepsilon)=\frac{N_{o}}{\varepsilon_{g}} \varepsilon ; \quad 0 \leq|\varepsilon| \leq \varepsilon_{g}$

Using the property of even function and substituting the expression of $N(\varepsilon)$ in the gap, we find

$\frac{1}{\lambda}=\frac{2 T_{c}}{\mathcal{E}_{g}} \ln \left[\cosh \left(\frac{\varepsilon_{g}}{2 T_{c}}\right)\right]+\int_{\mathcal{E}_{g}}^{\omega_{c}} \frac{1}{\mathcal{E}} \tanh \frac{\varepsilon}{2 T_{c}} d \mathcal{\varepsilon}$

where, $\lambda=N_{o} g$ is the measure of electron-boson coupling constant.

The integration in (4) cannot be carried out analytically. Thus, we are left with using approximation and numerical methods. The integrand function is so peculiar that it is very difficult to reasonably approximate it with other function over a large interval. Using numerical integration, the $\lambda-T_{c}$ plots showing the effects of gap $\varepsilon_{g}$ and cut-off energy $\omega_{c}$ are shown in Figs. 3a and 3b, respectively.

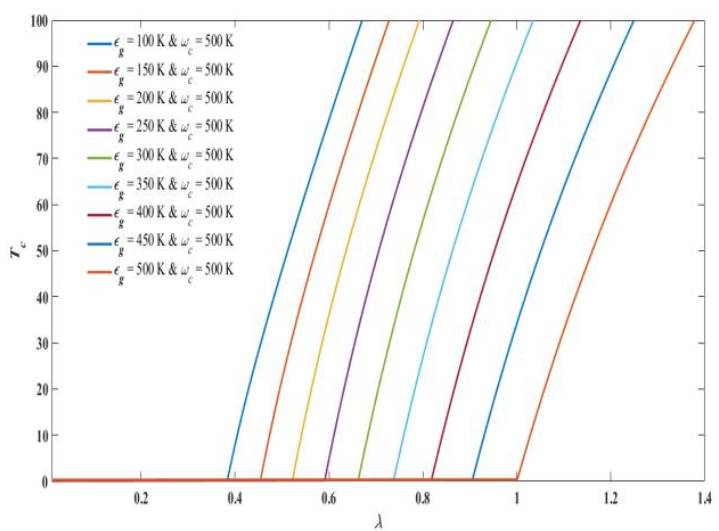

Fig. 3a. Variation of $T_{c}$ with $\lambda$ for different gap values $\varepsilon_{g}$.

Fig. 3a shows that as the gap approaches the cut-off, the electron-boson coupling $\lambda$ required for the same $T_{c}$ gets larger meaning the non-superconducting orders suppress the superconducting order. 


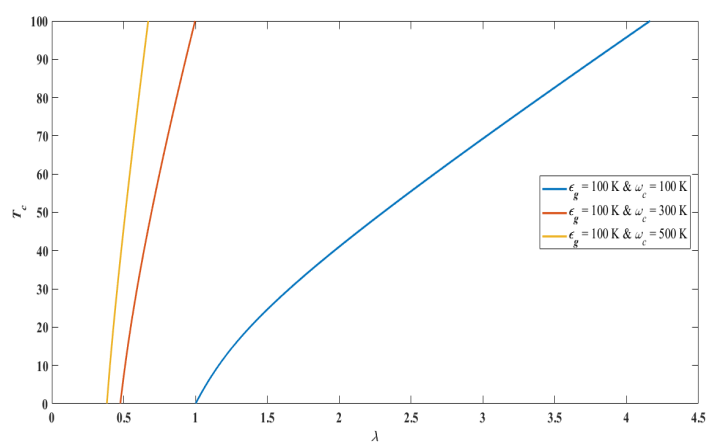

Fig. 3b. Variation of $T_{c}$ with $\lambda$ for different cut-off values $\omega_{c}$.

Fig. $3 \mathrm{~b}$ shows that as the cut-off is increased, the picture is opposite to that of Fig. 3a: electron-boson coupling $\lambda$ required for the same $T_{c}$ gets smaller. Also, $T_{c}$ increases at a faster rate with $\lambda$ in accordance with the BCS picture.

There is a special case, $\varepsilon_{g}=\omega_{c}$, for which there is an exact and completely analytical solution. In this situation the EDOS will be as shown in Fig. 4 below.

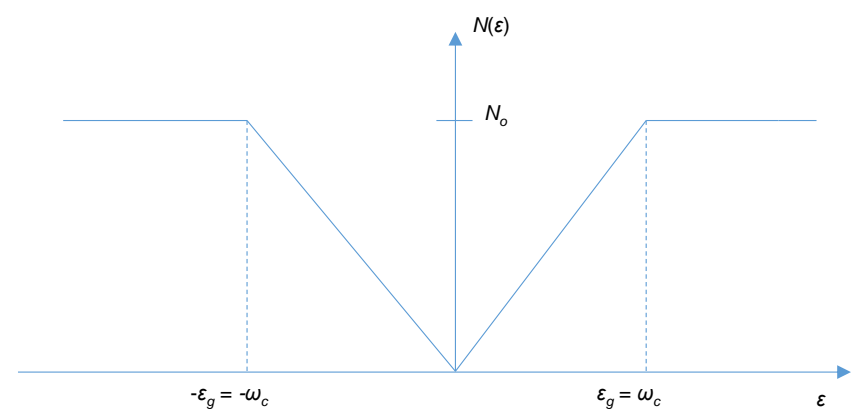

Fig. 4. EDOS in the special case, $\varepsilon_{g}=\omega_{c}$.

In this case we will have the (implicit) expression for $T_{c}$

$\frac{1}{\lambda}=\frac{2 T_{c}}{\omega_{c}} \ln \left[\cosh \left(\frac{\omega_{c}}{2 T_{c}}\right)\right]$

The corresponding $T_{c}-\lambda$ plot along with the BCS $T_{c}-\lambda$ plot is shown in Fig. 5 .

It is seen that solution in this special case yields non-zero $T_{c}$ only when the electron-boson coupling constant is large, in agreement with the feature revealed by Fig. 3a. We also see that the larger the cut-off energy the sharper is the increase of $T_{c}$ with $\lambda$ similar to the BCS case, again confirming the characteristic shown by Fig. $3 \mathrm{~b}$. The increase of $T_{c}$ with $\lambda$ becomes extremely sharp when $\omega_{c}$ is large in the strong 
coupling regime. This is due to the combined effect of the gap being equal to the cutoff energy and increase in the cut-off energy. This extreme steepness can be indicative of electronic instability.

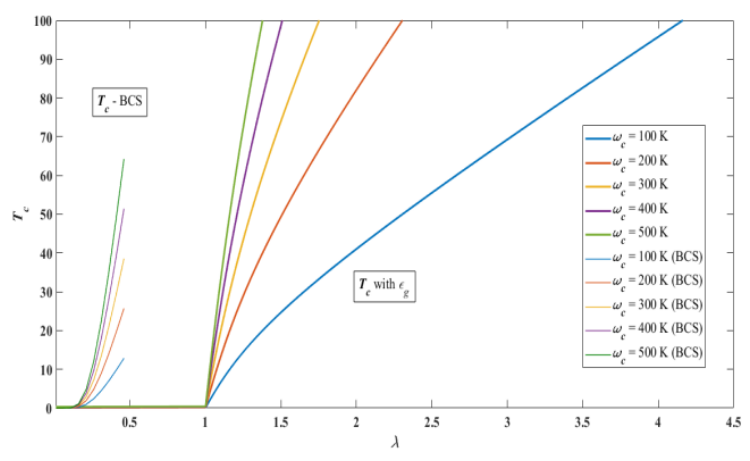

Fig. 5. Variation of $T_{c}$ with $\lambda$ with and without PG for different values of $\omega_{c}$.

Let us now consider the case where the non-vanishing gaps affect only the EDOS as shown in Fig. 2 (we are assuming $\varepsilon_{g} \leq \omega_{c}$ ), averaged over the entire energy window. There is no contribution to the energy of a Cooper pair $[11,12]$ in the integrand. We will have the $T_{c}$ equation

$$
\frac{1}{g}=\int_{0}^{\omega_{c}}<N(\varepsilon)>\frac{1}{\varepsilon} \tanh \frac{\varepsilon}{2 T_{c}} d \varepsilon
$$

giving

$T_{c}=1.13 \omega_{c} e^{-1 / \lambda_{\text {red }}}$

with

$\lambda_{\text {red }}=\lambda\left(1-\frac{\varepsilon_{g}}{2 \omega_{c}}\right)$

Since we have taken, $0 \leq \varepsilon_{g} \leq \omega_{c}$, we find, $\lambda / 2 \leq \lambda_{\text {red }} \leq \lambda$. Thus, we have a reduced coupling constant. The variation of the ratio of the $T_{c}$ given by (7) and the BCS $T_{c}$ with different PG energy scale is shown graphically in Fig. 6 . We see that in this case, $T_{\mathrm{c}}$ decreases with PG. The decrease in $T_{c}$ continues almost linearly with increase in PG until the vicinity of the cut-off energy is reached where the variation is a bit rolled-off.

We have seen that presence of PG in the EDOS manifests itself by suppressing $T_{c}$. Now let us have a look at a real system, namely, $\mathrm{YBa}_{2} \mathrm{Cu}_{3} \mathrm{O}_{7-\delta}(\mathrm{Y}-123)$. We will consider the variation of the superconducting transition temperature $T_{c}$ with $\mathrm{PG}$ temperature $T^{*}$ or equivalently PG magnitude, $\Delta_{\mathrm{PG}}$ (we are assuming $\Delta_{\mathrm{PG}}=k_{B} T^{*}$ ), for pure Y-123 $[13,14]$. We compare this with the $T_{c}$ depression curve of previous section. The comparison is shown in Fig. 7. 


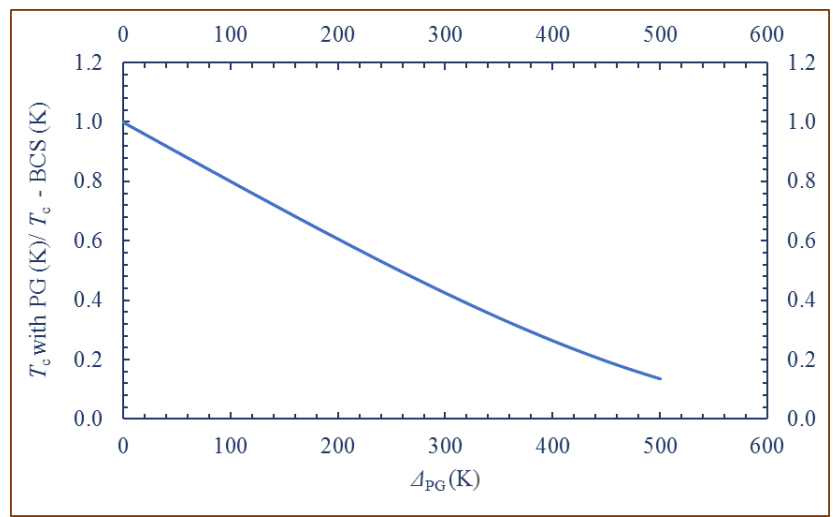

Fig. 6. Variation of the normalized transition temperature with PG energy (expressed in temperature scale).

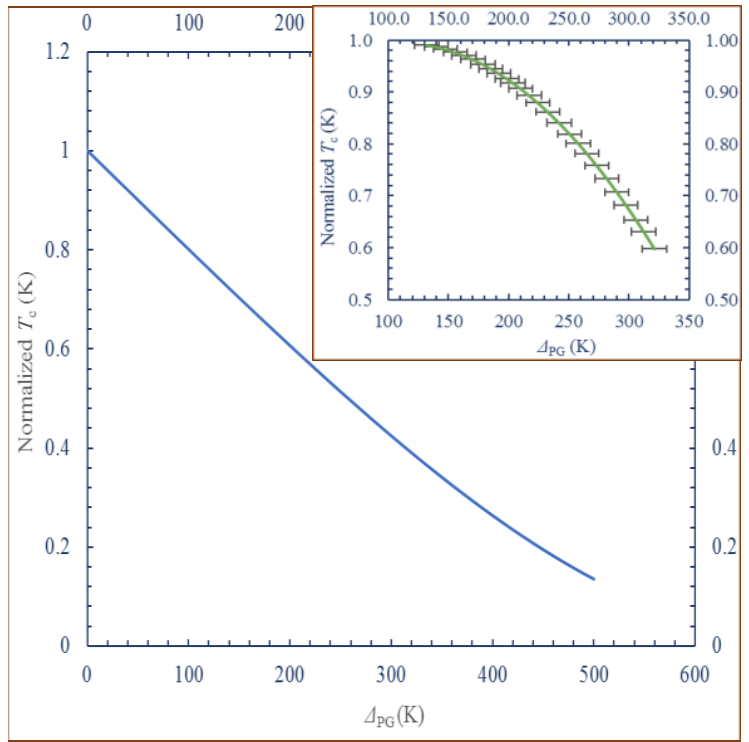

Fig. 7. Normalized $T_{c}$ as a function of PG energy scale. Main: Theoretical. Inset: Experimental variation of normalized $T_{c}$ with PG energy $[13,14]$.

We see that the pattern of variation of $T_{c}$ is similar in both the cases except that the roll-off of the curve occurs at small values of PG for the Y-123 system and for our calculated scenario the roll-off occurs when the PG approaches the cut-off energy. Though the axes of the plots are similar for both the graphs but still they are not plotted on the same graph because the cut-off energy for the theoretical plot has been selected arbitrarily, whereas $\Delta_{\mathrm{PG}}$ of Y-123 are experimentally estimated. 


\section{Discussion and Conclusions}

BCS gap equation [15] considers only one type of gap in the EDOS, the superconducting gap, which vanishes at $T=T_{c}$ but in some unconventional superconducting systems such as hole doped cuprates, there may appear PG, CDW gap, and SDW gap, which do not necessarily vanish at $T_{c}$. We have investigated the effects of these gaps on $T_{c}$.

The integral BCS formalism [15,16] works well with superconducting gap for weak-coupling limit i.e. $\lambda<1$. But in presence of non-vanishing gaps we find that, the more ubiquitous the gaps are in the EDOS, the stronger electron-boson coupling is required to back up superconducting order. From this we get hints that nonsuperconducting gaps restrain superconducting order. When the cut-off window is widened, SC persists at lower electron-boson coupling.

In the special case $\varepsilon_{\mathrm{g}}=\omega_{c}$ (Fig. 5), SC exists in the strong coupling regime but also the superconducting order builds up very quickly with electron-boson coupling. This happens because we are considering $\varepsilon_{g}=\omega_{c}$, so that, not only the non-superconducting gap but also the cut-off is changing. This indicates that natural systems with nonvanishing gaps can have strong superconducting order parameter and hence high $T_{c}$. But this will also require large non-vanishing gap values, meaning strong order of the corresponding type. As the gap gets smaller very strong electron-boson coupling will be required, making such system difficult to realize practically.

In the general case with non-vanishing gaps, solving the $T_{c}$ equation ultimately reduces to an integration which cannot be integrated analytically for arbitrary limits. Analytical method can be applied only for certain limits but arbitrary limits can be tackled with the help of numerical integration. But two tricky features are extracted from our analysis: when the non-vanishing gap is quite smaller than the cut-off energy, $T_{c}$ is suppressed in the sense that large coupling is required compared to the BCS scenario while if the gap approaches the cut-off, though SC exists in the strong coupling regime, but $T_{c}$ increases very abruptly with the coupling.

The simple analysis in which the effect of non-vanishing gaps has been considered only on the EDOS, $T_{c}$ simply decreases with gap. Furthermore, the form of depression of $T_{c}$ closely resembles that of the experimental situation for Y-123 [13,14] except when the gap is too small or $T_{c}$ itself is high. When the $T_{c}$ is high, near the optimum doping, the PG temperature and the onset of strong superconducting fluctuation come close together. Therefore, $\Delta_{\mathrm{PG}}$ cannot be located precisely. This introduces error in experimental estimation of $\Delta_{\mathrm{PG}}$. For this reason, the $\Delta_{\mathrm{PG}}$ values are possibly overestimated when $T_{c} \sim T_{c}$ max . Therefore, this difference may arise from experimental artifact.

The analysis carried out in this short communication is incomplete. We have used weak-coupling BCS integral expression to obtain $T_{c}$-equations under different situations. To generalize the formalisms developed here, we wish to extend the analysis incorporating strong coupling. 
We also intend to solve the superconducting gap equation with realistic SDW and CDW gaps with their respective temperature dependences in future.

We must also mention that none of the $T_{c}$-equations used here takes Coulomb pseudopotential into account. This repulsive term always reduces the value of the superconducting transition temperature. For completeness, this term needs to be taken into consideration. We intend to do so in a future study.

\section{Acknowledgments}

The authors are grateful to Raihana Shams Islam, Department of Physics, Rajshahi Univrsity, for her co-operation in the earlier phases of the research.

\section{References}

1. J. R. Waldram, Superconductivity of Metals and Cuprates (IOP Publishing, Bristol, 1996).

2. M. H. Hamidian, S. D. Edkins, C. K. Kim, J. C. Davis, A. P. Mackenzie, H. Eisaki, S. Uchida, M. J. Lawler, E. -A. Kim, S. Sachdev, and K. Fujita, Nature Physics 12, 150 (2015).

3. M. R. Presland, J. L. Tallon, R. G. Buckley, R. S. Liu, and N. E. Flower, Physica C 176, 9 (1991). https://doi.org/10.1016/0921-4534(91)90700-9

4. I. Kokanović, J. R. Cooper, S. H. Naqib, R. S. Islam, and R. A. Chakalov, Phys. Rev. B 73, 184509 (2006). https://doi.org/10.1103/PhysRevB.73.184509

5. S. H. Naqib, and R. S. Islam, J. Supercond. Nov. Magn. 27, 337 (2014). https://doi.org/10.1007/s10948-013-2303-4

6. S. H. Naqib, Supercond. Sci. Technol. 20, 964 (2007). https://doi.org/10.1088/09532048/20/10/012

7. J. W. Loram, K. A. Mirza, J. R. Cooper, W. Y. Liang, and J. M. Wade, J. Supercon. 7, 243 (1994).

8. J. L. Tallon, R. S. Islam, J. Storey, G. V. M. Williams, and J. R. Cooper, Phys. Rev. Lett. 94, 237002 (2005). https://doi.org/10.1103/PhysRevLett.94.237002

9. B. J. Ramshaw, S. E. Sebastian, R. D. McDonald, J. Day, B. S. Tan, Z. Zhu, J. B. Betts, R. Liang, D. A. Bonn, W. N. Hardy, and N. Harrison, Science 348, 317 (2015). https://doi.org/10.1126/science.aaa4990

10. J. L. Tallon, and J. W. Loram, Phys. C 349, 53 (2001). https://doi.org/10.1016/S09214534(00)01524-0

11. S. A. Kivelson, I. P. Bindloss, E. Fradkin, V. Oganesyan, J. M. Tranquada, A. Kapitulnik, and C. Howald, Rev. Mod. Phys. 75, 1201 (2003). https://doi.org/10.1103/RevModPhys.75.1201

12. L. N. Cooper, Phys. Rev. B 104, 1189 (1956). https://doi.org/10.1103/PhysRev.104.1189

13. S. H. Naqib, J. R. Cooper, J. L. Tallon, R. S. Islam, and R. S. Chakalov, Phys. Rev. B 71, 054502 (2005). https://doi.org/10.1103/PhysRevB.71.054502

14. S. H. Naqib, J. R. Cooper, J. L. Tallon, and C. Panagopoulas, Physica C 387, 365 (2003). https://doi.org/10.1016/S0921-4534(02)02330-4

15. J. Bardeen, L. N. Cooper, and J. R. Schrieffer, Phys. Rev. 108, 5 (1957). https://doi.org/10.1103/PhysRev.108.1175

16. J. Bardeen, L. N. Cooper, and J. R. Schrieffer, Phys. Rev. 106, 1 (1957). https://doi.org/10.1103/PhysRev.106.162 